\title{
Decision-making in Shiatsu bodywork: complementariness of embodied coupling and conceptual inference
}

\author{
Michael Kimmel ${ }^{1}$ (D) Christine Irran $^{2}$
}

Accepted: 4 December 2020/ Published online: 1 February 2021

(C) The Author(s) 2021

\begin{abstract}
"4E" cognitive science has demonstrated that embodied coupling offers powerful resources for reasoning. Despite a surge of studies, little empirical attention is paid to discussing the precise scope of these resources and their possible complementariness with traditional knowledge-based inference. We use decision-making in Shiatsu practice - a bodywork method that employs hands-on interaction with a client - to showcase how the two types of cognitive resources can mesh and offer alternative paths to a task: "Local" resources such as embodied presence, empathy, attunement, as well as skilled perception-action coupling are not only central for implementing a successful therapeutic intervention. The immediate coupling with a client also offers basic means of deciding about fitting and meaningful interventions. Yet, when comprehensive intervention strategies are at stake, Shiatsu decision making must be complemented through "non-local" resources, notably inferences rooted in anatomy/ physiology knowledge, categories, heuristics, and mental models. To draw out implications for "4E" cognitive science, we argue that "local" embodied coupling and "nonlocal" conceptual inferences can functionally complement, inform, and scaffold each other in a dialectic process.
\end{abstract}

Keywords Bodywork · Decision making Embodied reasoning $\cdot$ Interactivity $\cdot$ Conceptual inference $\cdot$ Cognitive integration

\section{Introduction}

What happens as a body engages with the world (things, spaces, or people), according to old-style Cartesian cognitive science lies outside cognition proper; it is a delivery

Michael Kimmel

michael.kimmel@univie.ac.at

1 Cognitive Science Hub, University of Vienna, Vienna, Austria

2 Vienna, Austria 
system for the mind and a system of action implementation. In contrast, a set of approaches within "4E" (embodied, embedded, extended, and enactive) cognitive science (Robbins and Aydede 2009) have successfully demonstrated that perception and action within the socio-material realm can facilitate reasoning, decision making, and creativity (Kirsh 2014; Steffensen et al. 2016). Emphasis has equally been put on the participatory genesis of meaning between agents (Di Paolo and DeJaegher 2007; Fuchs and DeJaegher 2009) and the role of coupling dynamics (Araújo et al. 2006; Dumas et al. 2014; Silberstein and Chemero 2011). Some would even locate cognition in transactions, the "in-between" of agent and ecology (Ihde and Malafouris 2019; Steffensen 2013). In line with this, cognition is considered a form of adaptive action in the world (Engel et al. 2013) instead of "mind-bound" thinking after the Cartesian model. In our present context, we summarize these as views of embodied-enactive, ecological, and interactivity-based (EEI) theories, whose critique of cognitivism takes a fundamental form, e.g., enactivism seeks an analysis of "minds in terms of dynamic patterns of adaptive environmental interactions" (Ward et al. 2017, p. 372).

To date, EEI scholarship and more "disembodied" theories of conceptual reasoning remain at loggerheads. But more importantly, their explananda themselves seem somewhat disconnected, almost as if each type of theory best explained a particular phenomenon. As EEI approaches to skills strive to avoid the appeal to "internal" resources, they often make their case with smaller-scale illustrations based on dynamic systems modeling (e.g., Baber et al. 2019), minimal simulations (Di Paolo et al. 2008), simplified experimentation (e.g., Walton et al. 2015), or anecdotal analysis (e.g., Kiverstein and Rietveld 2018). Such EEI approaches may run up against serious problems in modeling complex expert skillscapes (Kimmel 2017), in which immediate perception-action coupling is combined with multiple other resources. At present, EEI theory seems to leave a phenomenological gap open to the extent that the first-person viewpoints of experts, even when they report numerous perception-action skills (e.g., Gesbert et al. 2017; Kimmel and Rogler 2019; Macquet 2009; Poizat et al. 2013; Seifert et al. 2013), are equally rich in subjective concepts most easily assimilated to the theoretic language of mental models (Steiner et al. 2017). To name but a few desiderata, future EEI approaches should respect the functional (and usually also didactic) separation of technical skills from decision making capabilities, model improvisation repertoires, address expert resources such as imagery, abstract principles, or metacognitive reasoning, as well as explain how all this draws on a "dynamic, multidimensional knowledge base" (Jensen et al. 2000).

These problems speak to the scaling-up problem of explaining "higher-order" cognition in terms of sensorimotor interactions (Di Paolo et al. 2017; Gallagher 2017). More generally, EEI scholars face the challenge of so-called "representationhungry" tasks (Clark and Toribio 1994), which result from the need to reason about hidden, future, or counterfactual aspects, and which are typical for many professional skills. In particular, it remains unclear how sophisticated decision making and knowledge-based inference can be accommodated within EEI theories. It is thus fair to say that embodied-interactive research calls for integration with research on expert concepts and their use in practice. One neglected avenue towards harmonization, which we propose to explore at present, is to place dynamic coupling and inferential reasoning mechanisms in a single analysis in order to ask how they become mutually supportive in meshworks of intersecting practices, to borrow Ingold's (2015) seminal concept. Our 
principal aim in this paper is to demonstrate how to approach the analysis of such meshing processes.

Our showcase is a bodywork method ${ }^{1}$ known as Shiatsu, which blends the power of embodied immediacy with sophisticated reasoning tools. When we started researching Shiatsu we immediately became attracted to EEI theory, yet soon also came to realize certain practical limits: How practitioners find a fitting treatment is not only grounded in the continuous intercorporeal encounter with the client, but also massively shot through with conceptual operations. Shiatsu teachers will underwrite that presence, empathy, attunement, as well as perceptual and technical abilities are essential, and that refined intercorporeality skills are needed. Yet they equally emphasize the importance of studying Eastern medical theory for effective diagnostic assessment and for finding a good strategy. In Shiatsu training novices must laboriously memorize and learn to apply feature lists, principles, reasoning heuristics and network models of a semantically rich and tremendously abstract appearance. Consequently, calling Shiatsu an art of embodied interaction seems to be a half-truth of sorts; it is fundamentally an art of combining resources of different kinds in contextsensitive ways - which is why the domain makes for an excellent test-bed for cognitive theory.

In view of the ianus-faced competency set of Shiatsu, we will set out to discuss diagnostic and strategic decision making as regards (a) the scope of immediate coupling- and affordance-based resources, and (b) the meshing and integration of such resources with knowledge-based inference and concepts. E.g., physiology knowledge or strategy heuristics are recruited at many points, yet guided by - and taking effect within - embodied interaction. In other words, we claim that Shiatsu decision making unfolds at the intersection of "local", coupling-based resources with various "nonlocal” resources, to borrow and slightly adapt Steffensen's (2013) terminology.

\section{Shiatsu}

Our present discussion condenses findings from a micro-phenomenological ethnography of Zen Shiatsu, a direction of Shiatsu that puts strong emphasis on interactionbased strategizing and improvisation. Our study was based on 18 interviews with advanced learners and six with experienced practitioners, each of 2-3 hours duration, which reviewed specific interactions with clients; we complemented this through structured self-observation during Shiatsu practice by C.I. and another collaborator, Christina Regorosa. Our aim was to generate a competency profile of Shiatsu experts. The involved competencies include a multitude of perception, manipulation, and interaction skills, but - importantly - also diagnostic and strategic capabilities. Bringing the latter into focus is our present aim. Before we begin, our readers will benefit from some domain background.

\footnotetext{
${ }^{1}$ Bodywork includes osteopathy, physiotherapy, Feldenkrais, Rolfing, and many other approaches. In many countries these have the status of complementary medical therapies; in other places such as Austria, where our data was collected, the accepted term is "practice".
} 


\subsection{Background, aims, and philosophy of health}

Shiatsu is rooted in the Japanese massage/acupressure tradition (Palanjian 2004; Robinson et al. 2011). In a Shiatsu treatment, the practitioner uses fingers, palms, elbows, knees, or feet to implement acu-pressure techniques, massages, stretches or mobilizations. An attentive and sensitizing kind of touch is employed, with the aim to regulate the client's energy systems. Complaints addressed in Shiatsu include physiological issues like fatigue and insomnia, headache, muscle and joint pain, menstrual or menopausal issues, edema, susceptibility to infection as well as psychological distress. A typical session may take $40 \mathrm{~min}$ or longer and takes place with the client lying on a mat with his or her clothes on.

Shiatsu sees health as a dynamic equilibrium. Body, psyche, sociality and lifestyle are understood as integral factors of a complex self-organizing system. Its philosophy is to stimulate the client's self-regulating capabilities, adaptiveness, and resilience. ${ }^{2}$ Hence, instead of following a mechanistic approach that focuses on local leverage, a dispositional re-organization of the whole system is sought. Accordingly, practitioners are taught to think systemically and multi-causally. They consider themselves enablers who encourage and invite transformations from various angles, but respect the client's self-organizing healing process; frequently they adopt a playful, probing attitude, use gentle transformations, and take multiple, possibly indirect routes.

In implementing this philosophy, Shiatsu practice focuses on re-harmonizing the client's energetic system. With major loans on Traditional Chinese Medicine (TCM), Shiatsu adheres to the therapeutic principle that the vital energy known as Qi/Ki (Liu 2018), which plays a key role in the regulation of living organisms, needs to be restored to its natural, balanced state. Shiatsu shares this approach with acupuncture, Tuina, and other schools or Eastern medicine, as well as historically less related notions such as Indian prana. The underlying assumption is that living beings source Ki from food and breathing, process it, convert it into usable energy, or stockpile it. Ki contributes to sustaining and coordinating musculoskeletal mobility, metabolic activity, nourishment of tissue, and mental-emotional balance.

In keeping with this framework, Shiatsu practice interprets a client's ailments as corresponding to specific dysfunctional "energy constellations" (Porkert and Ullmann 1982). The harmonizing techniques used by Shiatsu practitioners focus on patterns of energy blockage, imbalance, or problem chaining as well as mutually sustaining patterns of $\mathrm{Ki}$ excess or depletion. To find and assess such dysfunctional energy constellations, specific models were adapted from the TCM tradition, which focus on twelve Ki pathways known as Meridians and associated Functional Circuits (Beresford-Cooke 2003). These notions originate in classical texts and in handeddown knowledge of systematically observed correlations between (a) Ki activity in particular body areas and on particular pathways, and (b) specific psycho-physiological symptoms/complaints (a more detailed explanation will follow in later sections). The state of Meridians and Function Circuits is believed to be, in part, perceiveable from

\footnotetext{
2 The philosophy of Shiatsu and related medical systems is remarkably affine to complexity-theoretic views, which focus on self-organization in complex adaptive systems, be they biological or (inter)personal - in the bodywork case they are both (Weiss et al. 2003; Bell et al. 2012; Koithan et al. 2012; Pincus 2012; Pincus and Metten 2010; Fogel 2013; Tschacher et al. 2003; Tschacher et al. 1998).
} 

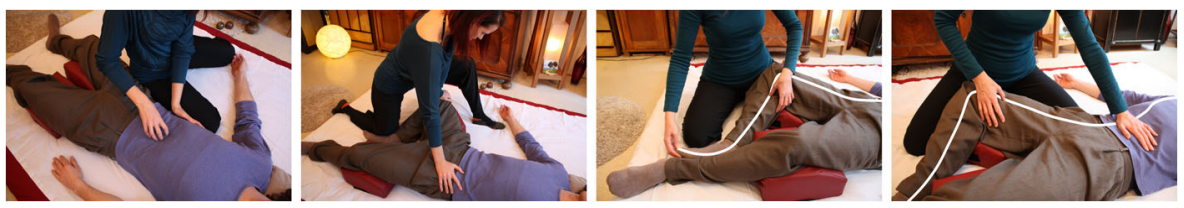

Fig. 1 [from left to right]: a evaluative palpation, $\mathbf{b}$ preparatory release routine, $\mathbf{c}$ and $\mathbf{d}$ acupressure along a Meridian indicated as white line

the body surface; Shiatsu practitioners thus learn to ascertain the energetic profile of these structures through skilled forms of touch (see examples in Fig. 1).

Techniques that harmonize energetic imbalances are believed to improve ailments manifesting in many other aspects of physiology such as metabolism or sensorimotor functions. Since Ki is held to govern a person's self-organizational capability as a whole, its appropriate regulation can trigger system-wide effects. Some Shiatsu schools add functional anatomy techniques to energy harmonization, which address muscles, joints, tendons, and fascia similar to those an osteopath or physiotherapist might use (Normann 2020).

Importantly, the vital energy $\mathrm{Ki}$ is not merely a theoretical concept; it extends into the practitioners' sensate reality who learn to apply manual pressure to stimulate, collect, or scatter the Ki running through the Meridian, often by acupressure (Palanjian 2004). In addition, joint and muscle mobilizations may be used to foster free Ki circulation or a balanced Ki distribution. Unsurprisingly, the ability to perceive and influence energetic phenomena is a fundamental prerequisite for giving Shiatsu. The feeling of $\mathrm{Ki}$ is, e.g., described by practitioners as tingling sensations, warmth, flow properties like streaming, or subtle force sensations comparable to magnetic attraction or repulsion. ${ }^{3}$ Shiatsu givers learn to differentiate Ki qualities across body locations or tissue types, and recognize distinct forms of $\mathrm{Ki}$ actualization (notably hypo- and hyperactivity) as well as distinct Ki distribution patterns.

\subsection{Participatory sense-making, intercorporeality, skill}

Despite our present focus of the "giver" it is crucial to understand that a Shiatsu treatment operates in a genuine dialogic mode (Kimmel et al. 2015). Shiatsu is a prime example of a coregulation (Fogel 1993) and participatory sense-making process (Di Paolo and DeJaegher 2007; Fuchs and DeJaegher 2009), in which the continuous adaptive coupling via bodily cues (e.g., touch, gaze, movement, postural change) enables agents to regulate their interaction. ${ }^{4}$ Practitioners attune their breath with the client's, notably to be able to provide pressure in sync with the client's breathing. They immediately adapt their pressure intensity and action rhythm to the clients' muscle tone, stress level, and attentional state. A

\footnotetext{
${ }_{3}^{3}$ Although electromagnetism and skin conductivity are likely involved (Becker et al. 1976; Oschman 2000), we do not concern us here with what Ki "really" is or whether it exists as integral phenomenon from a natural science viewpoint.

${ }^{4}$ In EEI approaches, frequent reference is made to mechanisms of primary intersubjectivity such as embodied empathy and resonance, rhythmic or affective attunement, shared attention, and perception-action coupling (Trevarthen 1979). These mechanisms, which are characteristic of face-to-face contexts, have an operative intentionality of a fundamentally embodied kind, as the other's intentions are manifest in her appearance and actions, such that "[m]y own intentions are operable [....] within the perception of the other's intentions" (Gallagher 2017, p. 80). On the other hand, in more secondary forms of intersubjectivity, including narrative ones, may add to this (Øberg et al. 2015), as evidenced by verbal diagnostics in Shiatsu.
} 
bodily resonance loop is established, which, throughout the session, allows practitioners to immediately feel which invitations the client readily accepts, remains indifferent to, or resists. Practitioners possess a specific skill that allows their hands to perceive and actively address the interior structures of the client.

It's hard to overemphasize how much the therapeutic intervention in Shiatsu thrives on the ongoing intercorporeal encounter with a client. Practitioners incorporate the client's feedback and adaptively respond to the unfolding process through touch- and empathy-based presence. To accentuate what this means, this continuous participation in the client's process contrasts sharply with an acupuncture treatment where needles are set after diagnosis and the client is left alone for the effects to unfold. Shiatsu follows a much more participatory approach which is appreciated by many clients because it allows practitioners to optimize techniques, embrace serendipity, and react to challenges "on-the-fly". A further benefit is that status assessment and strategic reasoning - functions at the higher level of decision making - can be refined through the process itself, as we shall see.

Despite this continuous reciprocal adaptation the encounter isn't symmetric. It is the professional responsibility of the practitioner to guide what happens. The role distribution is in some respects like in tango or salsa dancing where one partner leads, but where followers have some power to set the emphasis, assimilate or reject input, request a slower pace, etc. The "leader", the Shiatsu expert, can sensitively respond to the client's arising needs, while the client answers "invitations" or expresses requests through verbal feedback or in more subtle ways through the autonomic nervous system, changed muscle tone, or changed breathing. To the expert, groans, relaxing sighs and bowel sounds can indicate changes in the nervous system (e.g., entering a parasympathetic state); perceived temperature changes or changes in skin color indicate activation states; and whether a client participates is indicated by whether he or she moves along or relaxes into stimulations (as opposed to holding patterns, stiffening, or twitches) as well as through open or closed eyes.

When Shiatsu practitioners engage with the client they cultivate a respectful, patient, and receptive attitude. They emphasize the therapeutic importance of presence, trust, and creating a safe space. These factors heighten the client's participation, "open" the senses, and ensure the optimal assimilation of inputs (Kimmel et al. 2015). Importantly, embodied co-presence is employed as a tool. Practitioners use their own relaxed breathing, a calm resonant voice, mindful touch, and continuity to positively affect the client's well-being (see Blackburn and Price 2007). The immediacy of intercorporeal rapport is used to influence the client's regulatory system in two complementary ways. On the one hand, it helps practitioners to find the right intensity and quality of stimulation, notice incipient changes, and respond immediately. On the other hand, intercorporeality offers the client a "platform for self-experience". It provides them with a resonance medium that allows a more reflexive access to somatic processes, enables clients to expand their adaptive capabilities and explore new behaviors (e.g., improved movement patterns facilitated by the practitioner). Overall, these general intersubjectivity related skills benefit what is commonly called a good therapeutic alliance; they provide unspecific effectiveness criteria (Haken and Schiepek 2010) of a therapy, the "smoothers" and "enablers" of a client's systemic self-organization.

In addition to these intersubjectivity related skills, practitioners must acquire a broad range of specific techniques for mobilization, toning, and stimulation and must master general principles, such as exerting pressure from the body center with one's weight, 
instead of muscle force. By a similar token, a broad range of active perception techniques must be mastered, such as palpation to distinguish tissue impairments (bloated vs. swollen; tense vs. stiff) and specific mobilizations to ascertain if a joint is blocked or decentered. In the interest of brevity we refer readers to Kimmel et al. (2015) for a skill overview.

While all this implies years of training, it only specifies how to implement techniques. The mastery of intersubjectivity skills and the technical toolbox does not yet tell the practitioner what to deploy when. This brings us to the complex issue of therapeutic decision processes, i.e. on what basis a practitioner selects and combines techniques, while determining how and how long to execute them or when to adapt or even replace them. EEI approaches tend to assume that these decision skills are as coupling-based and embodied as technical skills, yet it remains a weighty theory question to what extent they deserve this epithet.

\subsection{Decision making in Shiatsu}

A general structure is common to most Shiatsu sessions, which constitutes the frame in which more specific decisions are to be made. At the outset the client reports an issue and is interviewed about its history. The Shiatsu practitioner asks about injury, medication, stress, diet, sleeping habits, lifestyle, and how complaints manifest in everyday activities (cf. Monteiro and Norman 2013). This allows contextualizing impressions collected later during the treatment. The actual physical interaction starts with a phase of attunement, e.g. by synchronizing breath while resting one hand quietly on the client's abdomen. A systematic check of the main energy sub-systems of the body is then carried out, which draws on information gathered via palpation, for example of dorsal or abdominal reflex zones, combined with observational data such as perceived body alignment.

Once practitioners have concluded their first diagnostic assessment their manual therapy can begin. This consists of multiple modules, which should fit together for good combined effect. A number of functional complexes of the body are typically addressed, for example activating selected Meridians via acupressure, combined with joint mobilizations and myofascial releasing techniques. A before-after comparison may ensue before ending.

Within the discussed procedural constraints, a treatment strategy may either arise step-by-step as the practitioner goes along, follow some approximate (!) thematic focus decided early in the session, or a mix of both. Kimmel et al. (2015) contrast different degrees of strategic constraint, where some decision making scenarios leave much open for later interactive specification, while others impose greater constraint in advance. However, no Shiatsu treatment is fully decided one-shot. Strategies can, to an extent, be developed, refined or augmented within the interaction process. Practitioners want to ensure context-sensitivity even after the initial check; a session thus proceeds throughout in an "implicitly diagnostic" mode. While initial checks, verbal reports, and previous sessions constrain all that follows, there is always flexibility for decisions made underway.

In this respect, decision making in Shiatsu runs counter to much therapeutic cognition literature, which tends to dichotomize assessment and treatment ("you diagnose, then you implement"). Both, pattern recognition and hypothetico-deductive accounts of diagnostic assessment (Klemme and Siegmann 2015) remain anchored 
within a cognitivist framework. The deeply embodied and interactive nature of the reasoning process is overlooked, even if combinations of inference and search are described in this literature (Croskerry 2009a, b; Custers 2013; Edwards et al. 2004; Grace et al. 2016; Arocha et al. 2005; Lawson and Daniel 2011; Monteiro and Norman 2013; Norman et al. 2007; Patel et al. 2012; Patel and Ramoni 1997; Sibbald and de Bruin 2012; Zhang et al. 2004). Scarce attention is paid to recursive perception-action coupling and decision resources that emerge when actively engaging with a client. In contrast, Shiatsu clearly underwrites more interactivity-based and enactive views which have recently emphasized that assessments are recursive and interwoven with intervention (Kimmel et al. 2015; Normann 2020; Øberg et al. 2015). Øberg and colleagues term this embodied-enactive clinical reasoning. A closely related idea is that practical reasoning happens in ways that make a therapy a "contextualized interactive phenomenon" (Higgs and Jones 2000, p. 5; cf. Jensen et al. 2000). This resonates with the wellknown concept of reflection-in-action (Schön 1991), where knowledge is developed in and through action. Hence, practitioners do not only implement prior decisions about apt procedures, but genuinely reason within the encounter.

If we give interactivity and real-time responsiveness their due without throwing out informed strategic awareness, we need new ways to talk about intentions. It is undeniable that many therapists project ahead macro-procedural templates of a session or mention "ballpark" ideas about possible strategies. Yet many details may be left open or strategies kept flexible. Speaking about plans and goals in the traditional sense may not be very helpful (Suchman 1986) ${ }^{5}$; instead we can speak of forms of non-deterministic intentionality that constrain what is to come without fully defining it. To go beyond plans and goals EEI theory has postulated directives which specify directions of search (Engel 2010; Davis et al. 2015). Similarly, improvisation science has proposed notions such as sketch planning (Norgaard 2011), framing intentions, effect-range anticipations, contingency plans, and action horizons that embodied improvisers use to remain flexible (Kimmel et al. 2018).

A further difficulty bears mention with respect to decision making in a bodywork context. Different decisions across a session need to be treated as interdependent (a) since the conditions for upcoming decisions emerge from how the system responds to prior decisions, and (b) because every decision must respect the interaction history, make sense cumulatively, and allow closure at the end of the session. This kind of task constellation is known as a dynamic decision making (Brehmer 1992; Gonzalez et al. 2005, 2017; Hotaling et al. 2015). Since, treatments emerge in a fashion that must respond to changed problem states the process is never predictable, yet things must add up at the end.

\section{Embodied-interactive decision making}

Our aim in this section is to discuss how the immediate interaction between practitioner and client, the "local" functions of perception and action, contribute to decision making.

\footnotetext{
${ }^{5}$ Decision-making is hierarchical, from local tactical to strategic issues - the former ones often concern how to best implement an already selected technique, how long to continue it, or how to flesh out some detail or other. At the local level somewhat "ready-made" procedures may be found, but very seldom at the higher level.
} 


\subsection{Affordances and interactivity-based reasoning}

The growing emphasis on interactivity in scholarship of therapeutic reasoning reflects concepts from general EEI theory, where cognition is regarded as a form of action-inthe-world. To EEI views, intelligent behavior arises from dynamic coupling with the ecology; they emphasize how much power lies in active engagement, as opposed to "internal" reasoning in a mental locus.

Presently, we will discuss the Shiatsu-related scope of two central EEI ideas from the fields of ecological dynamics and interactivity theory, respectively. The question represented by the ecological dynamics approach (Araújo et al. 2006, 2017; Esteves et al. 2011; Fajen et al. 2009; Passos et al. 2012) is how tactical decisions are regulated through perceived affordances. It has been shown that in team sports complex forms of coordination can be created in this fashion (Silva et al. 2013). For instance, a defensive configuration of the opposing team provides real-time indications of how to orchestrate an attack by one's own team (Passos et al. 2011), and creative synergies can be improvised on-the-fly in this way (Kimmel et al. 2018; Torrents et al. 2016). In these cases the involved individuals draw on "local" information to discern possible doables and select those fitting the task and their current pursuits. Such regulation resources operate through unmediated sense-act loops (see Dawson et al. 2010). Ecological information provides guidance for action in real time.

The second question is associated with the interactivity-based approach (Cowley and Vallée-Tourangeau 2010; Kirsh 2009, 2014; Kirsh and Maglio 1994; Steffensen 2013; Steffensen et al. 2016; cf. also Malafouris 2013). It concerns cognitive benefits that accrue from active exploration, manipulation, feedback stimulation, interactive solution probing, and guiding the attention of others. It is claimed that cascading processes of give-and-take in the world come to leverage cognition. This implies that reasoning happens transactionally and through the coupling itself, an idea recently applied to clinical reasoning (Pedersen 2015; Trasmundi and Linell 2017; Trasmundi and Steffensen 2016). The locus of cognition is not the mind alone. Experts - through skilled interaction - can partly "offload" mental tasks and exploit features of the dynamic for this purpose.

\subsection{Real-time tactics and strategies}

A first level of Shiatsu decision making indeed stays close to the embodied level of coupling and exploits the immediacy of the intercorporeal dialogue, which operates through the "resonance loop", as we termed it. Since a practitioner's strategies are seldom pre-determined fully or for good, to what extent is decision making possible through the process of embodied interaction itself?

First off, the immediate coupling has the benefit of mutual reactivity. Practitioners can pick up relevant information from the client, say pressure sensitivity, expressions of pain, stagnant breath or sighs, muscle twitches, or heat flashes, and use this to adapt their tactics. They can directly perceive "neediness" and react to warn signals immediately, e.g. when they overstimulate an aching area. The interpersonal coupling allows for sensitive adaptations of duration, intensity, or even of the preferred tool for specific treatment modules. When the client's system suddenly starts to reorganize one can accompany this process at once. One can also reduce physical pressure when signs of 
discomfort arise, calm the patient, etc. Hence, at the tactical level, how proximal aims are best reached often suggests itself through sensitive real-time coupling and the affordances it generates at each moment.

At this tactical level, the claims of interactivity theory equally find validation in Shiatsu, with respect to how reasoning is facilitated through active engaging. It is demonstrable that practitioners exploit embodied interaction for their reasoning. Frequently, they start with unspecific, but beneficial interventions which will likely generate further clues, e.g., by relaxing the client's system, stimulating overall Ki flow, or deblocking joints. Seeing a body function respond to input may be helpful to understand its coordinative interplay with other functions, e.g. a mobilization may clarify if a particular muscle "tags along" with a muscle group it should not normally be involved in. Practitioners thus capitalize on stimulated feedback (Kirsh 2014) and solution probing strategies (Cowley and Vallée-Tourangeau 2010; Steffensen 2013). Furthermore, thanks to interactivity one need not pick a strategy "one-shot". Selecting among affordances can occur while acting (Cisek and Kalaska 2010); many practitioners start to flesh out strategies once they receive more information (dynamic specification in Kimmel et al. 2015). To sum the scope of interactivity-based mechanisms up, they are used to optimize ongoing interventions, to specify tactical means and their duration, and to elaborate or revise a diagnosis.

The obvious next question is whether this embodied reflection-in-action can play out beyond tactical matters. Can it shape decisions about what type of intervention should follow next? The answer is a qualified "yes": There is evidence for a basic strategic approach in which the practitioner browses body areas for issues that "cry out for attention" (such as energy imbalances or inhibitors to energy flow), alleviates these locally, and moves on. Practitioners can thus follow "the trail of symptoms" as they move along. Such ad hoc strategizing proceeds such that (a) they actively scan for further therapeutic affordances once a module is completed, or (b) the client's reaction to the last intervention reveals another "needy" area, a process that Gaver (1991) terms sequential affordances. ${ }^{6}$ The next choice can thus be guided by information collected during the previous intervention. Often it is the client's observed response to a specific technique that suggests further aspects of a problem configuration - for example new inhibitors may become clear, so that the practitioner adds a treatment module or a preparatory technique. A strategic trajectory can thus emerge on the basis of multiple affordances when an improvised approach is aimed at.

\subsection{The scope of "local" decision making}

Decision making based on affordances exemplifies behavioral regulation as EEI theories conceptualize it. The emphasis lies on embodied coupling and the momentby-moment guidance it provides. Thus, how to sequence and weigh modules is not decided in advance, but emerges as one goes along. As we have seen, the benefit is responsiveness to unexpected leverage points and the ability to embrace serendipity

\footnotetext{
${ }^{6}$ In addition, indirect benefits for decision making accrue from sensitive intercorporeal coupling. Since highquality coupling provides support for the client, it can encourage affordances, especially by making things the client finds uncomfortable more acceptable and by inducing readiness for change.
} 
when a reactive zone pops up on-the-go or when some "neediness" grabs one's attention. Also, when unforeseen issues, errors, or sudden insights occur, a practitioner can immediately respond.

This kind of ad hoc approach to developing a fitting strategy, however, also shows limitations if it is the only resource a practitioner uses. The underlying therapeutic assumption of ad hoc strategizing is that removing multiple blockages can already trigger self-organized transformations in the client's system; thus a sufficient amelioration will unfold by adding up local interventions. The empirical question is to what extent this assumption holds: Experts frequently consider this a relatively non-holistic approach. They typically say that, even when the serial alleviation of local problems may be beneficial, it may lack comprehensiveness or may overlook client sensitivities not apparent at face-value.

Readers may wonder why EEI decision making models, e.g. from ecological dynamics, can work well in sports, dance, and martial arts, but show these limitations in therapeutic contexts, such as Shiatsu. Consider the contrast with soccer: Here, affordances are subject to a cumulative logic within a particular attack: one can only shoot a goal after a series of passes, etc. Thereafter, the game logic is "set back to zero" and previous events at best matter psychologically. Setbacks earlier in the game can be corrected later. The dynamic of soccer is thus moderately pathdependent, considered over the whole $90 \mathrm{~min}$. A Shiatsu session may not be so forgiving, since it is strictly path-dependent throughout: (a) modules should make sense with respect to one another; (b) earlier introduced modules may impose constraints on later ones, and (c) a treatment must make sense as a whole as well as allow functional closure. Hence, when practitioners pick a technique they must factor in the interaction history and prospective constraints to ensure global effectiveness (and thus a projected direction, however minimal). Not all affordances available from a momentary viewpoint also make sense in the larger picture. One must filter among them. Thus, any Shiatsu treatment must make sense as a whole, implying that the practitioners may have to reason about compatibility of parts.

\subsection{Taking stock \& preview}

To sum up this soccer analogy, the critical issue is how much an approach purely based on local assessment can guarantee an effective mutual calibration of treatment modules and a good session closure. An even greater challenge to EEI arises when practitioners aim at integrated interventions of a systemic kind. The theoretical crux is not that affordances cannot be based on information from highly complex and relational information arrays (see Runeson 1977). Nor does the problem relate to the fact that a holistic diagnosis requires temporally extended and recursive explorations.

Rather, we shall argue that affordances as such may offer too little integrality unless a theory-informed information search is done or unless anatomical imagery, categorical inferences and heuristics are used in conjunction with affordances. The crux is that, in the context of a therapy session, surface appearances of a client may be too shallow or even misleading. What is salient is not necessarily what is important. The surface appearances may allow weighing the information in quite different ways, or even turn out as being unreliable when they do not reflect hidden and long-term causalities. As a 
consequence, reasoning mechanisms that are based on the immediate embodied coupling frequently need to be "assisted", and in challenging cases literally "augmented", through conceptual inferences (a claim further discussed in the concluding section).

As a roadmap for the reader's orientation, Fig. 2 contrasts basic forms of EEI strategizing, a form of purely embodied sense making that we term ad hoc strategizing (left), with a more comprehensive strategizing path we call conceptual-embodied sense making (right), which the next sections will explain and discuss. We see here different paths a practitioner may choose and what they involve in terms of cognition.

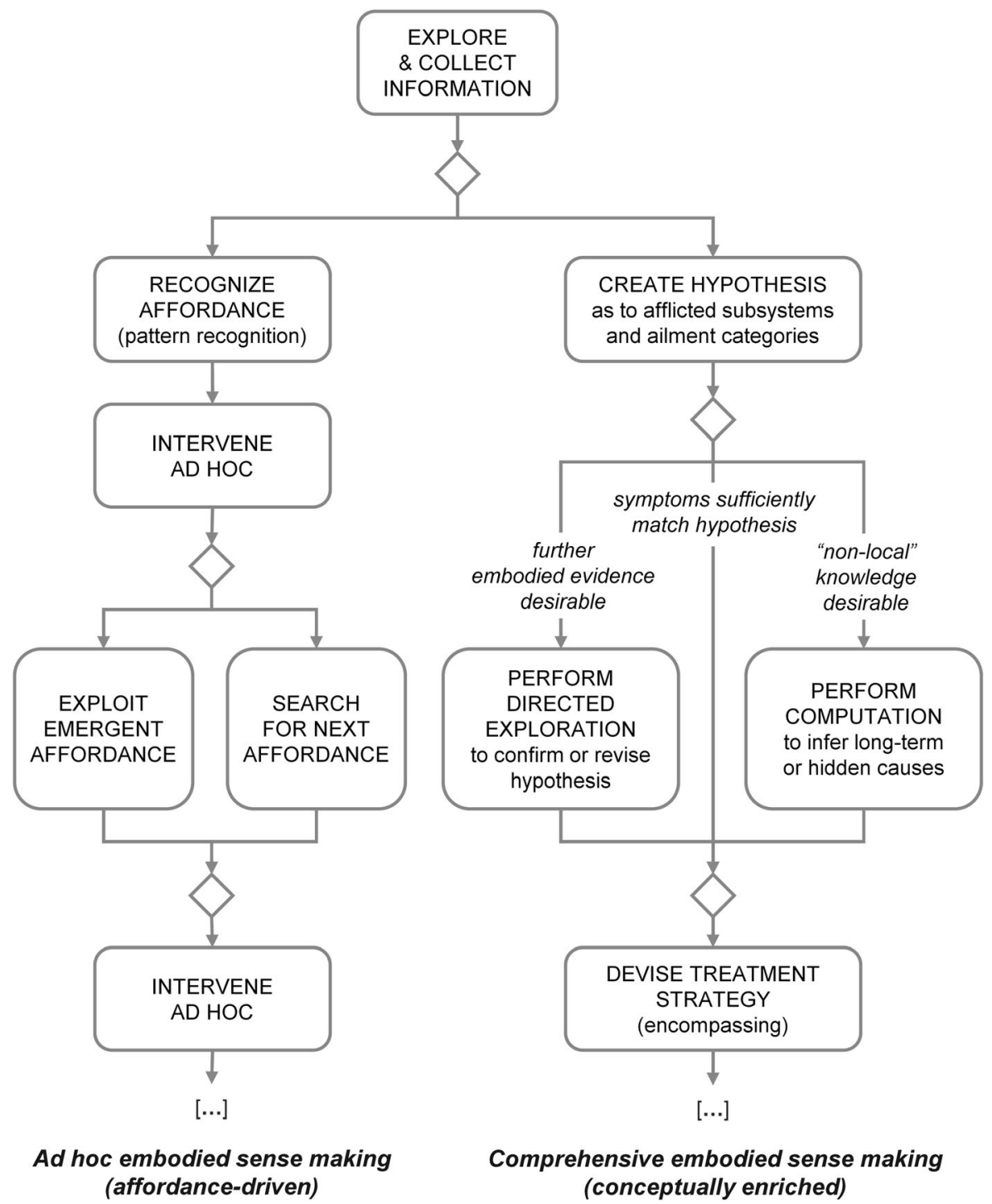

Fig. 2 Schematic of therapeutic decision paths and resources 


\section{Advanced decision making tools}

Shiatsu practitioners, even when they make the most of affordances and interactivity, benefit from consulting "non-local" resources, which allow for more sophisticated decision making processes.

\subsection{Embodied holism: systemic perception and structural knowledge}

At its root, a strong demand for "non-locality" ties in with Shiatsu's historically grown holistic philosophy of health, which was previously described. Let us now take a closer look at several perceptual and cognitive resources that this approach entails.

Holism implies sensitivities for the body as a systemic network of relations. Shiatsu practitioners are trained in a genuinely systemic evaluation of the client. While novices still train to identify local energy signatures, which are described through the terms Jitsu (excess) or Kyo (depletion), advanced practitioners understand these local signatures as part of a systemic whole and summarily seek to discern wider patterns of Ki distribution and flow (see Herfel et al. 2007; Hsu 1999). Let us specify what a systemic assessment means in the Shiatsu context: The client's health profile is ascertained against the yardstick of a model, in which the meso-scopic energy profile of various physiological functions should be balanced (i.e. neither excessive nor under-active) and in which the macro-scopic profile should display smooth and well-adjusted interplay between sub-systems. This overall gestalt-like appearance of Ki activity is regarded as a key to a systemic health evaluation. On this basis, practitioners single out for treatment sub-systems that appear to be out of kilter. ${ }^{7}$

To evaluate gestalt patterns in a client, precise general knowledge of the human body is so fundamental that it usually even informs the simplest strategic scenarios. This resource is known as structural knowledge (Schoppek 2002) in cognitive approaches to the regulation of complex adaptive systems and refers to knowledge of available network connections in a particular system. In Shiatsu, this concerns anatomical and physiological topologies, connective pathways, and what functional components need to be distinguished. For example, practitioners treat spots on a single Meridian, a pathway running across the body, as part of one integral structure because they are known to share certain energetic qualities. Besides manifest structures, this knowledge may shade into more abstract network conceptualizations of systemic functions, which we will discuss in more detail later.

Structural knowledge frequently appears before the mind's eye as a grid of connections on a body map, which Shiatsu trainees internalize. Phenomenologically, this implies experience-near forms of imagery in which properties known from anatomy

\footnotetext{
${ }^{7}$ When the interplay is healthy, the sub-systems show responsiveness to stimulation and bidirectional reactivity (e.g., Ki redistributes to an adjacent subsystem). Specific imbalance patterns, in contrast, are seen as indicating dysfunctions, which can manifest as compensatory or otherwise disturbed interplay, as disproportionally active or isolated subsystems. This logic implies energetic correspondences, e.g., excessive $\mathrm{Ki}$ is held to compensate for a corresponding hypo-active area. A similar logic applies to the movement apparatus, which focuses on relations such as muscle agonist-antagonist pairs or tensegrity structures in fascia networks (Silva et al. 2010). Anomalies may manifest as postural asymmetries, encumbered ranges of motion, or compressed inner organs (e.g., due to stress on the diaphragm or psoas tension).
} 
textbooks or previous experience "augment" the practitioner's actual sensations. Not only do practitioners imagine hidden tissue layers running below the skin, they also imagine specific connective relations. The actual perception of the client is therefore seeped with anatomical and physiological imagery and potential patterns of interplay, which practitioners, for lack of a better expression, "read into" surface features.

Latching such structural imaginations onto a kernel of perceived features allows more targeted information search, for example by guiding the hands along a connective anatomical pathway. Hence, EEI theory - despite its apparent neglect of structural imagery - will be happy to hear that a body map of this sort is, inter alia, cultivated for its interactivity related benefits. It licenses specific exploration and stimulation practices that clarify if body components communicate, show disproportional activity, or are locked in some dysfunctional interplay. To wit, when exploring a client, practitioners report conceptualizing horizontal relations of interplay between areas (or sub-systems) as well as hierarchical notions about emergent "ensemble" functions, i.e. synergies between parts (Kimmel et al. 2015). Practitioners use this structural knowledge to guide their attention in various different ways: They may follow a muscle chain to check if impulses propagate as they should. They may also check if two potentially associated body areas co-vary in their reactions or stimulate them simultaneously to reveal if they communicate enough to create an ensemble function (e.g., to distinguish problems in components from problems of how components communicate). Often, practitioners will also explore if a local stimulus reverberates throughout the body-system or gets "smothered" somewhere.

However, structural knowledge, in due course, also performs a mediating function for more complex interpretive operations: Over a treatment many system relations of a client come to be explored, which, by and by, add up to a macro-scopic impression. Structural knowledge supports this process by organizing and fusing all gathered information into coherent holistic meanings. Furthermore, since a body map allows perceptual indicators to be localized, yet also to see the whole, the systemic logic of an issue comes to be conceptualized. Practitioners might, for example, speak of "stagnancy" of "arrested" Ki on a specific Meridian (the canonical metaphors used for this purpose) or express the gist of the client's dysfunctional condition in terms of a "vicious circle" or "problem propagation" between two or three sub-systems. Hence, practitioners summarily interpret a client's network dynamics in terms of effect propagation, condensations, and blockages on particular pathways in this physiological configuration.

\subsection{Assisted embodiment: reasoning with pathogenetic categories, principles, and heuristics}

To identify comprehensive problem constellations several other cognitive resources can be invoked. Of which kinds these are depends. In the simplest of scenarios a practitioner just aims to alleviate several local imbalances as described in the section Realtime tactics and strategies. In a number of other treatment scenarios, a practitioner desires a more comprehensive treatment strategy. For this purpose, a global-relational profile of the client's problem constellation is created.

To illustrate the process, let's go back to the point when a session starts. The task of the practitioner is to look for signs and indicators, interpret them in context of the 
client's narrative or previous sessions, and bring them into a diagnostic logic based on experience of similar cases and theoretical knowledge. This proceeds by predicating the found problem constellation onto a canonical ailment category. In other words, practitioners must cluster and reference verbalized complaints, the client's appearance, and perceived energetic imbalances to the most fitting category of ailments.

In the classification model of Shiatsu, one of these category-types is comprised of (relatively comprehensive) regulatory networks called Functional Circuits combined with their energetic states. For example, a particular condition might be found that is labeled as "Weak Kidney Ki". The term "Kidney" refers to a Functional Circuit (named after its principal organ), ${ }^{8}$ while the term "weak" refers to its current Ki-state of depletion. Whenever sets of symptoms can be dominantly referenced to the dysfunctional state of a particular regulatory network such as the Kidney Circuit, a number of fitting intervention strategies can be inferred on this basis. Note, however, that performing this mental operation is demanding and requires training. Practitioners have laboriously memorized from lists and examples how sets of symptoms correlate with ailment categories. We may speak of correlational knowledge, which enables practitioners to slot the client's information with a category.

The actual process of classification during a treatment can involve different degrees of practical and cognitive complexity. To stick with our example, a "Weak Kidney Ki" constellation can be strongly suggested even by the initial appearances, whereupon a practitioner will inquire after other possible associated symptoms such as lower-back pain, sensitivity to cold, reduced stress resilience, fatigue, or anxiety, and verify the felt quality of Ki through palpation. If the majority of symptoms can be slotted with the Kidney Circuit (weighed against symptoms that might fit dysfunctions elsewhere, say, of the Spleen or Liver Circuits), this clarifies the therapeutic strategy, namely to strengthen the Kidney Circuit.

This first scenario leads to a relatively straightforward systemic diagnosis. How complex is the underlying cognition though? EEI theories might argue that the involved category knowledge is just a fancy way of talking about complex pattern recognition, a "smart" form of perception (Runeson 1977). While we have seen that this cannot be true with respect to the learning process which is semantically mediated through feature and mapping lists, in experienced practitioners this interpretation may be accurate enough, once perception has been automated and clusters simply "spring into view". Even with an assessment that stays fuzzy at first, practitioners may simply check for further diagnostic pointers or weigh alternative possibilities while they begin their first interventions. To some degree, diagnosis and its refinement is thus possible through interactionbased EEI principles in the case of Shiatsu experts.

In other cases, however, conditions are so demanding that EEI mechanisms alone cannot handle what happens. Instead, categorial reasoning needs to be used in real time in conjunction with EEI mechanisms, a process that has been repeatedly described in

\footnotetext{
${ }^{8}$ Although Functional Circuits carry organ names it is important not to reduce these to the material organ as biomedicine conceives of it; further structures and functions are involved.
} 
the clinical reasoning literature (Patel et al. 2005, 2012; Patel and Ramoni 1997). Let us assume the initial set of indicators that the practitioner finds appears ambiguous, selective, or has already led to an ineffective intervention. Such circumstances call for refining the assessment through a search and test procedure, which guarantees greater comprehensiveness and checks systematically for possible alternative interpretations. This involves what Patel and Ramoni (1997) have termed abductive reasoning - a process of refinement that combines hypothetico-deductive aspects with repeated, targeted exploration. In abductive reasoning, experts think through the lens of category models, which correlate clusters of indicators and symptoms with syndromes or pathogenic explanations. Often, the first set of impressions will suggest a possible category hypothesis. On this basis practitioners can check for possible further indicators associated with this category in a targeted way by running through the list of "usual suspects". This either confirms the hypothesized category or the accumulating data point to a different candidate. In the latter case, the initial hypothesis is replaced by a more likely candidate (recall again that indicators frequently cross-cut categories), which provides its own set of further indicators to check. The new hypothesis reorients the search direction and, by doing this repeatedly, a practitioner can generate an explanatory frame that accounts for symptoms parsimoniously.

In this way, conceptual knowledge of correlations between ailments $(=\mathrm{a}$ category) and indicators (= its instances) is combined with searching and testing. The use of categorial inferences thus mediates embodied-interactive processes and gives them direction. Evidently, this process is not merely "mind-bound". Rather, directed information searches and observing how the client reacts to distinct probes or stimulations alternate with categorial inferences. The overall strategic benefit of such a cyclic mediation process is easy to see: A body has far more indicators than can reasonably be explored within limited time and diagnosis invariably begins with incomplete data; in view of this an abductive process affords gains of explanatory depth.

Practitioners additionally constrain the process through a set of cognitive tools which help to detect non-salient information or causally related "players" of a dynamic. In this regard, our informants made frequent mention of simple principles or rules-of-thumb. For example, if a practitioner notices a spot with excessive $\mathrm{Ki}$ activity, he or she will often generate inferences from a diagnostic principle stating that "overactive $\mathrm{Ki}$ stems from a correlated $\mathrm{Ki}$ depletion elsewhere". The hypothesis of a correlated Ki deficit suggests scanning for tell-tale qualities in specific further areas and thus, again, expands the search in a targeted fashion. (Note that simple principles of this kind can support the most basic affordance-driven strategies that simply chain multiple local interventions.) Other, more comprehensive principles support the assessment of Ki distribution across several physiological sub-systems, such as the simple rule to "determine the most imbalanced [hypo- or hyper-active] Circuits in the system".

Principles for searching and checking frequently converge with actionstrategic principles, which share the same logic of energetic imbalances. This includes simple harmonizing principles such as "Where $\mathrm{Ki}$ has become excessive, distribute it", or complementarily, "where there is hypo-activity, stimulate Ki-accumulation". For the more comprehensive scenario discussed above, the associated action strategy is to "strengthen the weakest link" or "balance the 
most disharmonious Circuits". We even have evidence of complex heuristics as defined by cognitive scholarship of medical reasoning (Marewski and Gigerenzer 2012). These heuristics involve decision trees ${ }^{9}$ and contingency-based sub-routines such as "begin with treating Ki-empty areas to redistribute energy from excessive ones; if their over-saturation is too dominant (or stagnant) disperse it sufficiently before continuing". In such multi-stage procedures, the hierarchy of checks often begins with a default procedure such as "check for energy bottlenecks (as the most likely cause), if none stands out check for other causes".

In sum, when diagnostic or strategic inferences are made the Shiatsu system uses a set of rules-of-thumb, which both provide guidance for, yet also respond to interaction. Similar to what we noted earlier about abductive reasoning, they are interactively applied. In fact, the complex heuristics discussed last themselves specify how to combine simple embodied search and testing procedures with inference rules; they can be interpreted as templates for interaction-based reasoning. The reported resources thus combine the practitioner's general rule knowledge with interactivity-based mechanisms, which jointly effect the desired causal-explanatory refinement. In other words, heuristics and principles exemplify "non-local" resources designed for being used in interactively embedded ways.

\subsection{Augmented embodiment: diagnosing systemic causes with computational tools}

Shiatsu practitioners are frequently faced with situations in which a yet "bigger picture" is advisable, notably if they have reason to believe that initial appearances are diagnostically too inconclusive or even deceiving. For these cases, sophisticated reasoning devices exist, which pose the arguably most radical challenge to EEI principles because they are, in a relevant sense, symbol-manipulating computational devices as classical cognitive science defines them.

Shiatsu here draws on abstract network models originating in TCM, which operate at an abstract level and require a lot of training to link back to the physical reality of the client. These models supply tools for reasoning in complex or multi-causal problem constellations; they have historically evolved, based on empirical observations of Chinese doctors and their interpretation in different schools. Shiatsu practitioners notably apply these models when information is incomplete, conflicting, vague (e.g., when subtle compensatory patterns may have escaped notice or when there are scattered or inconspicuous anomalies), when risk factors from the client's narrative suggest an especially cautious approach, or when a systemically interwoven condition requires multi-causal therapeutic leverage. Abstract network models are also used when non-linear or chronic illness patterns are suspected or if a default approach stalls and a fall-back strategy is needed, e.g., to identify further Meridians that are afflicted by a chronic energy deficit (see example below). Shiatsu practitioners assume that using

\footnotetext{
${ }^{9}$ Heuristics have been defined as mental shortcuts that can be rapidly applied and that reduce information complexity. Marewski and Gigerenzer (2012, p. 82) state that "fast-and frugal trees are built around three rules; one that specifies in what direction information search extends in the search space (search rule); one that specifies when information search is stopped (stopping rule), and one that specifies how the final decision is made (decision rule)."
} 

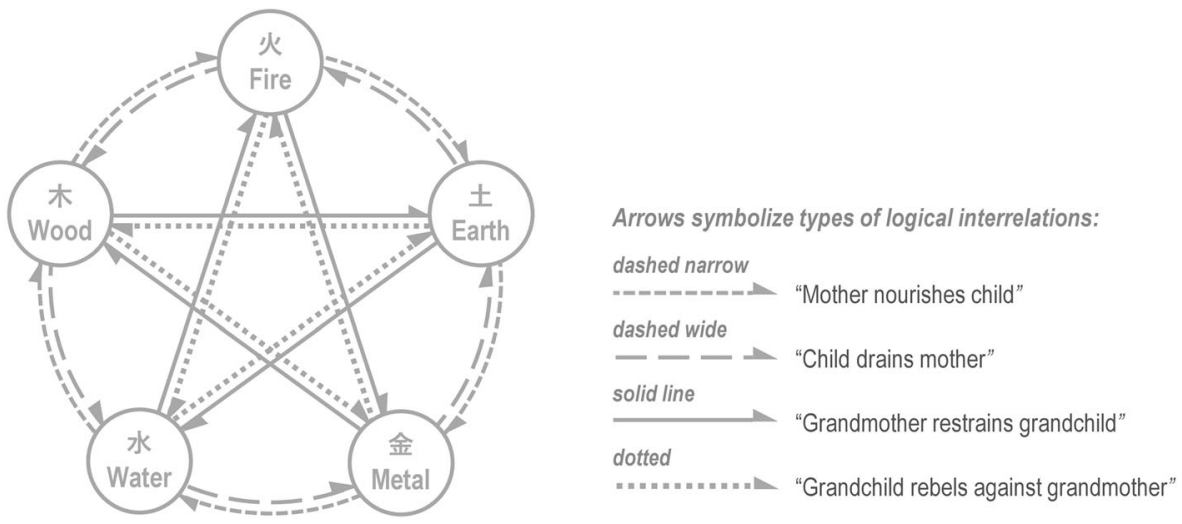

Fig. 3 Network model used for diagrammatic reasoning (Transformation Cycles)

network models helps to generate a comprehensive diagnostic profile, which justifies its cognitive costs.

It will help to provide a sketch how such models are used to compute deeper systemic causes of the client's condition: One common model, named the Transformation Cycles, helps to inquire into relational dependencies between sub-systems such as problem propagation paths or one sub-system blocking another's effectiveness. This model defines a network of "checks and balances" between sub-systems, which can "nourish" and "incite", "restrain", "fight", or "deplete" each other. This assumption is commonly summed up visually as five nodes arranged on a circle with an inscribed pentagram (Fig. 3) - a diagrammatic, but in practice also internalized reasoning tool.

In the diagram, circular arrows denote relations between "Mother" and "Child" nodes while cross-connectors on the pentagram are known as "Grandmother-Grandchild" relations. ${ }^{10}$ The nodes themselves are referred to as the Five Phases/Five Elements, each of which "compresses" a set of the previously mentioned Functional Circuits. ${ }^{11}$ When hidden or multiple problem causalities are suspected, the diagrammatic connectors suggest possible dependencies with further body systems that may be implicated, yet may seem inconspicuous at first glance.

The following scenario may serve illustration purposes: Suppose an under-energized "Spleen Circuit" and hyperactive "Gall Bladder Circuit" have been confirmed through bodily indicators and the practitioner has unsuccessfully tried to resolve the imbalance by strengthening the Spleen (which should have in turn effected a dispersion of excess energy in the Gall Bladder) and additionally treating the Gall Bladder with down-toning techniques. On the part of the practitioner, the insufficiency of this direct strategy triggers the meta-strategic decision to probe connections with further sub-systems by consulting the Transformation Cycles model:

\footnotetext{
${ }^{10}$ Clockwise oriented arrows on the circle and pentagram symbolize beneficial interplay (nourishing or controlling influences), while counter-clockwise ones stand for detrimental impacts (subsystems depleting or "revolting against" others). Linkages are often metaphorically described, as "Water hydrates Wood", "Wood feeds Fire", or "Earth chokes Fire".

${ }^{11}$ The Five Phases are differentiated into twelve Functional Circuits named after organs such as Spleen, Lung, Stomach, Gallbladder, Bladder, Kidney, etc., which are associated with biopsychosocial functions and particular Meridians.
} 
- Initially, a mental mapping from the Functional Circuits to the Five Phases logic is required: The practitioner infers that, in the model, the Spleen Circuit belongs to the Earth node and Gall Bladder to the Wood node. The Wood node exerts a controlling "Grandmother" influence on the Earth node. Given the earlier observed energy states, the Wood can be said to exert excessive restraint on its "Grandchild", the Earth, hereby weakening it.

- The practitioner now checks for systemic dependencies among neighbors that could help in a more mediated fashion to down-regulate Wood and to assist the revitalization of Earth. The diagram's arrows help with this: Wood's dependencies can be traced back to the Metal sub-system, which canonically is held responsible for keeping in check Wood activity. Given this "Grandmother" function fails, this may be a previously unconsidered factor contributing to Wood hyperactivity. The practitioner thus hypothesizes that the Metal node, if weakened, could be part of the systemic problem constellation.

- To test this hypothesis, a mental check of earlier physical and reported indicators is run; they indeed corroborate a weakened Metal sub-system (which was originally not targeted because the Earth's more prominent depletion had been prioritized.) This finding also makes sense in terms of the current model view. All data points now appear as part of a wider triadic logic in which the (slightly less conspicuous) energy deficit of the Metal can be traced back to a massively depleted Earth, it's "Mother" node. The inference that connects them is that the latter has ceased to supply sufficient nourishment, which in turn diminished the control Metal can exert over Wood.

- The central task now is to find a strategy to strengthen "Metal". This new intervention focus is assumed to be capable of breaking the vicious circle between the various sub-systems.

To sum up our example, ${ }^{12}$ a practitioner starts from indicator clusters, takes note of the associated semantic categories, and runs them through the Transformation Cycle's algorithm to compute an inference to a systemic cause, which is verified through embodied indicators. A more holistic diagnostic profile and a more powerful intervention strategy emerge. In our case the relevant insight was that it is necessary to start with treating a sub-system that, in the first assessment, showed less salient indicators than others. Hence, by applying the algorithm the practitioner makes a detour, which moves into progressively greater abstraction and hereby widens the problem space, before reentering embodied interaction.

Although the computational algorithm is run purely in the mind, able practitioners use it as a companion to embodied interactivity. The model-based inferences are both subject to verification and used for re-interpreting previous information, whose relevance can change by applying a new theoretical lens. In a sense, the "hidden layers" of the algorithm can be verified when the practitioner observes effect ranges of the intervention; the algorithm can also be nuanced and complemented interactively. Thus, the output of network models is re-situated in the interaction context - these abstract models are nested in a larger system of practices that concretize and ground them. Since

\footnotetext{
${ }^{12}$ In actual reality, experienced practitioners may confirm their evaluation through cross-checks with further algorithmic models we have not discussed here. Hence, the reasoning process can be multi-perspectival and move between models as well.
} 
the models are inherently fuzzy, they also require good embodied practices to guard against diagnostic errors.

In conclusion, why would semantic networks have arisen in the first place as reasoning tools? The answer is that for scattered/inconspicuous patterns and causes that are hidden or lie far in the past it helps to resort to the "wisdom of generations" (Kimmel et al. 2015). Kavoussi (2007, p. 294) argues that these models evolved to deal with non-linear biological functions with complex dependency relationships. He speaks of a multi-parametric semantic network for "the structural recognition, classification, and generalization of complex patterns based on the aggregation of variable weights and relationship strengths alone." It is probably accurate to hail the networks as tools for systems thinking (cf. Assaraf and Orion 2009; Jacobson 2000; Jacobson and Wilensky 2006; Sterman 2000). They capture the dynamic complexity of biological selfregulating systems and respond to problem constellations that are otherwise intractable, multi-causal, or go back far in system history. The tools thus respond to a specific type of "wicked" problem which faces experts with ambiguity, variable problem appearance, dispersed symptoms, and delayed, buffered, or disproportional feedback - all facets that tend to undercut strategies close to the surface of embodied coupling.

Regarding EEI theory, the usage of semantic networks holds important clues about functional limits of direct embodied coupling for certain classes of regulation problems. These semantic networks seem to involve complex mediating states with the capacity "to set-up and manipulate inner models" (Clark and Grush 1999, p. 9) and represent the controlled events instead of just comprising controllers that directly transduce information into action. The used stand-ins fulfill all criteria for "representation hungry" cognition after Clark and Toribio (1994, p. 18f). In other words, the networks "compress" or "dilate" inputs, i.e. in moving from sensory input to action outputs some cognitive machinery is interposed that reformats the input.

\subsection{Keeping track, synoptic awareness, and meta-regulation}

We would now like to discuss a final challenge which is not only characteristic of therapeutic interaction processes of all kinds, but of many other professional decision contexts as well. Although EEI theory focuses much on real-time process monitoring it is missing a specific account of how complex processes are integratively tracked and orchestrated. One aspect was already discussed, i.e. that deciding about a current treatment module is constrained by past and expected decisions. Another aspect not discussed so far is the fact that a multidimensional information ecology necessitates complex integrational feats based on highly organized attentional and memory functions. The long and the short of it is that expert decision making requires what, in expertise research, is known as situation awareness (Endsley 1995), a term referring to a cognitive structure that allows you to track where you stand with respect to current tasks, but also to assess this with respect to the wider task context, relevant information from the past, as well as general domain knowledge. 
In this regard, Shiatsu practitioners mention monitoring the overall status of the client, the state of the interaction, the completeness of diagnosis, and where the intervention stands. Specifically, ongoing checks are undertaken to (a) track changes in the client, (b) evaluate the degree of task completion and progress made, (c) evaluate the quality and dynamics of the encounter, ${ }^{13}$ (d) remember parameters a client responds to well or dislikes, and (e) keep contingencies, risks, as well as time constraints in view. Shiatsu experts emphasize how their ongoing assessment integrates earlier information - the backdrop of prior sessions and the client's history - and makes sense of it with respect to what may come next. In effect, their therapeutic situation awareness reads the client's system as an evolving dynamic network; it tracks the interplay of functionally associated body components and its evolution over time. Finally, practitioners report consulting their memory to compare different "snapshots" of the client's condition and to track delayed effects while keeping possible triggers from earlier in mind.

Overall, the phenomenological data suggest that practitioners keep a kind of updatable synoptic model in mind, which integrates different aspects of the task into a systemic view and evaluates these integratively. This is, for example, crucial for the anticipation of contingencies when using potentially risky techniques. Evidently, a synopsis involves context-driven information selection, update, as well as integration functions. Cognitively, this suggests that a so-called situation model (Zwaan 1999) is created, an updatable, constantly enriched memory structure of one or several treatment sessions. It is arguably only possible to achieve this by using a kind of expanded memory system that is known as long-term working memory (Ericsson and Kintsch 1995), which skilled performers are known to use to keep track of an extended event and a large amount of information, including context. Through these mechanisms practitioners progressively integrate salient information about the client as well as about the situation and hereby create an enriched gestalt.

Therapeutic situation awareness seems affine to EEI thinking in two respects: (a) with respect to constant updating of the ecological system status and (b) with respect to multi-timescale information, where temporally more extended dynamics are monitored via "context logic" (Davis et al. 2015), such that the perceived context indicates what momentary perceptions are most relevant. Conceivably, the EEI account can explain how comparisons of present and past, delayed effect tracking, as well as anticipations of contingencies operate. However, EEI approaches are hard pressed to explain the integrative assessment of a system and its dynamics, especially since illness categories, anatomy knowledge, and case analogies can shape what is considered relevant in context. These integrational feats pose serious challenges that EEI theories yet need to respond to.

This brings us to another hallmark of expert reasoning, namely meta-regulative process management capabilities. A distinctly meta-regulative stance (Veenman et al. 2006) is involved in knowing "what to do when" (Jansson 1994). Shiatsu practitioners intermittently make decisions of a supervisory kind (Ajjawi and Higgs 2012; Higgs and Jones 2000; Jensen et al. 2000; Klemme and Siegmann 2015), which supply navigational inferences and checks at critical moments. Their meta-

\footnotetext{
${ }^{13}$ With respect to the dynamics of the encounter, practitioners also often mention recognizing different forms of dynamic change over time. They notice "healthy variability" patterns in the client, growing openness to change, transitional moments when the system reorganizes, etc.
} 
regulative stance involves multiple aspects: First, time constraints are kept in view, e.g., when a practitioner deems it best to leave a salient problem for the next appointment, because doing full justice to it might exceed the available time, and sending the client off "half-way" could do more harm than actually help. Checks also are made to ensure optimal resource management (cf. Cohen et al. 1996; MacIntyre et al. 2014) as well as supervising one's attentional focus. Furthermore, both cognitive load and cognitive strategies are evaluated ("How certain is my problem analysis?", "Is further verification needed?", "Is the problem granularity sufficient?"). This indicates when to engage in effortful critical thinking, especially in anomalous situations. Similarly, it helps to weigh costs and benefits of specific resources ("Do heuristics suffice?"; "Will further information gathering afford gains?"; "Is trial-and-error sufficient?"; "Is an improvised or more structured approach advisable?"). Finally, self-checks support error management ("Did I misidentify goals, use a deficient model of the problem, or choose the wrong strategy?"). In sum, meta-regulative and supervisory functions not only serve to track the treatment process itself and its constraints, but also help practitioners to stay aware of their own cognition, the chosen tool mix, and possible alternative resources by engaging in critical thinking about their preferences. This means that multiple problem spaces (Higgs and Jones 2000) are held in focus for an integrated evaluation, a problem EEI does not yet effectively address.

\section{Implications for cognitive science}

We have presently used the therapeutic interaction domain of Shiatsu as a test-bed for the scope of EEI theory claims and as a basis to argue for cross-talk between different schools of thought.

\subsection{Basic, assisted, and augmented embodiment}

Shiatsu treatments proceed as a participatory, intercorporeal process in which a practitioner's interventions cumulatively work towards an integrated effect, while also respecting emergent dynamics. The domain presents us with a range of possible approaches to decision making and numerous possibilities of combining these.

To sum up our main findings, immediate embodied-interactive coupling with a client is fundamental. A high-quality embodied encounter is the regulative baseline for everything else; it ensures immediate therapeutic presence, rapport, responsiveness, and context-sensitive coupling. Interactive coupling can also supply functions that Shiatsu practitioners utilize to make decisions, yet with specifiable limitations: On the one hand, interaction provides a basis for tactical issues like tool selection and process optimization, and to some extent also for strategies of an ad hoc sort (i.e. "following the trail of affordances"). On the other hand, interaction-driven approaches applied in isolation show limitations. When comprehensive and holistic treatment strategies are at issue practitioners will typically also resort to an integrated, targeted exploration of the client; they bring to bear inferences based on category knowledge and heuristics, mental models, as well as supervisory process awareness. 
On the basis of these observations, we propose several levels at which EEI mechanisms may need to be complemented: We have seen that experience-near structural knowledge constrains and directs virtually any use of ecological information and that heuristic concepts assist functions such as information search; we have also seen that concepts can augment embodied functions in yet more elaborate ways when ecological information is fed into computations; finally we have seen that meta-processes can help to synoptically organize and regulate the activities in a wider skill system. All these functions are, however, dynamically integrated with interactivity-based and ecological cognition.

Typologically, the various decision making resources discussed fall into Steffensen's distinction between local and non-local resources, based on whether the practitioner's information is intrinsic to the intercorporeal "here-and-now" or not. A slightly more refined distinction could run between (1) resources immanent to interpersonal micro-coupling like empathy, attunement, shared attention, and sensorimotor coordination, (2) resources drawing on extended coupling which bring into play the wider setting ("situation logic"), the client's narrative, previous interactions, and situation awareness, as well as (3) resources drawing on general structural knowledge (e.g., physiology), salient case memories, as well as heuristics, categories, concepts, and mental models with a general and more abstract character. The definitional basis for drawing the line between local and non-local is whether a resource used to make a particular decision originates from the situated interaction dynamics or from some more general aspect of professional experience.

\subsection{Functional textures \& dialectic scaffolding}

Even when it is analytically central, the local/non-local distinction can be functionally misleading: The resource categories frequently mesh and amplify each other in a single texture, if we will. As related research has noted, there is a dialectical relationship between various modes of reasoning and knowledge paradigms (Edwards et al. 2004) where "different sources of experience, expression, and knowledge are integrated through reflection-in-interaction" (Øberg et al. 2015, p. 250). Practical expertise integrates these into a "gestalt" of different cognitive components (Braude 2017).

We propose to speak of process-based tool coalescence in which embodied coupling dynamics and knowledge-based inferences scaffold (i.e. trigger, modulate, amplify, and constrain) each other. This process is reciprocal: Embodied interaction shapes which concepts a practitioner selects, e.g., to enrich or disambiguate the diagnosis. Conceptual inferences, in turn, loop back on embodied interaction and may, e.g., suggest strategically meaningful continuations or further diagnostic tests. Concepts therefore get enlisted as function of interactivity, as a way of constraining its dynamics, but also to produce synergies together with it.

Understanding how this works ultimately depends on a micro-analysis of decision events, which we leave for future studies. At least, we have briefly sketched how knowledge-based inference enters into dialectic interplay with ecological, interactive mechanisms. This braiding can be alternating, although in Shiatsu embodied interactivity most frequently continues in parallel while inferences are made.

Furthermore, we aimed to contrast reasoning modalities across different problem contexts. Non-local resources are increasingly turned to when a comprehensive holistic 
intervention is deemed necessary, in face of diagnostic complexity or ambiguity, e.g., when a client does not respond to simpler treatment, and when the genesis and wider context of a problem need to be factored in. In such cases, knowledge-based inference helps to narrow down the problem space.

\subsection{Ontologically distinct, processually integrated}

Regarding cognitive science, our discussion speaks to the current debates on how socalled higher-level cognition sits with dynamic, interactive, and sensorimotor aspects (Bickhard and Terveen 1995; Clark 1997, 2008; Eliasmith 2012; Grush 2004; Kirsh 1991; Ramsey 2007; Smith 2010; Wheeler 2005). Our discussion raises the issue of how to bring conceptual inference into the fold of EEI theory and illustrates some typical challenges that complex forms of expertise entail.

Our own position in this debate is that it may be salutary, for now, to refrain from unification agendas that would subsume every cognitive resource under "embodied" or "interactive". Although, at least in our example, embodied interactivity provides the processual substrate for all cognition we embrace an "ontologically distinct, yet processually integrated" default position regarding the different types of resources. We notably wish to hold apart general knowledge and experience from its situated application. Although we agree that only situated application manifests what knowledge really does, we also harbor little doubt that Shiatsu givers, similar to other professional experts, live in a world of general concepts and sedimented case memories that are meaningful prior to any treatment. These have historically evolved to support the interaction-based decision making, but the fact that some of the resources are also used in less interactive forms of complementary therapy such as acupuncture nicely accentuates their semi-independent status.

In Shiatsu, non-local resources are typically recruited as-needed to disambiguate diagnosis, guide intervention strategies, or choose context-sensitive tactics. We can thus think of concepts as not having any stand-alone purpose here; rather they are recruited in a "pragmatic" function (Engel et al. 2013). We also follow RaczaszekLeonardi (2009) who considers symbols as "constraints on dynamics", and Wheeler who holds that symbols nudge process dynamics (2005, p. 229), as well as Smith (2010, p. 337) who defines conceptual knowledge as dynamically emergent integration phenomenon of heterogeneous lower-level processes, in which subsystems educate each other in virtue of their functional couplings.

Furthermore, a dialectic view chimes with approaches that hybridize "offline" and "online" cognition: We notably concur with De Bruin and Kästner (2012, p. 552) that offline cognition provides vital resources for online processes. They emphasize that temporary decoupling "mainly serves recoupling: taking certain processes offline, decoupling allows for novel ways of relating to the environment that provide the agent with more or better tools to act on certain affordances or create new ones. This in turn enables the agent to re-enter into online processing." Related approaches envision mechanisms of "unclamping" the online coupling when situated resources are deemed insufficient (Davis et al. 2015), distinguish modes of coupling (Kimmel et al. 2018), or speak of higher-level internal sense-making (Torrance \& Schumann 2019). Note that when we apply this kind of conceptualization to bodywork domains, it is possible for a practitioner to go offline into a mental reasoning space, while the intercorporeal 
situation remains unbroken and a treatment continues. Merely the provenance of the decision resource changes.

Finally, in one way or another, knowledge-based inference inserts a third player in a basic perceive-act loop, a mediating instance that filters input information, re-organizes, or reformats it, e.g., through input compression or dilation after Clark and Toribio (1994). Readers may have noticed that, to avoid entering the philosophical fray, we have largely eschewed the loaded notion of representation with its problematic metaphor of "content" and the questionable issue of identifiable decomposition-based carriers of meaning, not to speak of the broader representationalist paradigm. We have resorted to the less embattled notion of conceptual inference, since how psychology conceives of concept application needn’t involve assumptions about "content” (Löhr 2019).

\subsection{Conclusions \& future directions}

In taking stock of the cognitive phenomenology of experts we have taken care not to reduce the sophistication of the skill system in question. This careful approach, in our view, is mandated to illustrate just how difficult it is to reduce higher cognition to a derivative of sensorimotor cognition and interactive coupling. Even in a domain where everything is in fact about interaction, critical differences between local and non-local resources meet the eye.

Functional complementariness, on the other hand, was shown to be central: Examples like Shiatsu point to multi-way mediation between local and non-local resources on a massive scale and we illustrated a systemic multiplex network of tools which can interpenetrate, modulate, and complement each other in shifting alliances and forms of coalescence. We take the view that this is characteristic of many professions in which continuous systemic process regulation is required and where local and non-local resources, quite naturally, become mutually mediating. All this seems to suggest specific strategies to put EEI theory to the test:

- Providing a comprehensive profile of what professionals need to function in their domain in naturalistic settings.

- Probing for limits of EEI mechanisms (cf. Kirsh 1991) to find out about task context and task profiles (such as complex dynamics) that make them needful of partner mechanisms.

- Focusing on how conceptual inferences and the power of embodied coupling mutually augment each other, while tracing how they arise within interaction, and trying to explain why detours into non-local functions benefit local coupling.

Ultimately, we hope these questions pave the way for cross-talk and help to broker between " $4 \mathrm{E}$ " and traditional cognitive science: An approach that tracks the dialectic mediation between local and non-local resources may help us to circumnavigate an "either-or" and to fathom common ground, while encouraging a careful process analysis of resource meshworks. Scholars should see the study of high-level expertise in therapeutic as well as other domains as a rich opportunity to learn about how different cognitive mechanisms mutually inform, constrain, or amplify each other. 
Acknowledgments We thank our expert and learner informants as well as Christina Regorosa, Katharina Sabernig, Ludger van Dijk, and Hillel Braude for discussing previous versions of this paper. We also wish to express our gratitude towards two anonymous reviewers, whose comments helped to improve this paper much. The underlying research was funded by the Austrian Science Fund (FWF) under project P-23067.

Funding Open access funding provided by University of Vienna.

Open Access This article is licensed under a Creative Commons Attribution 4.0 International License, which permits use, sharing, adaptation, distribution and reproduction in any medium or format, as long as you give appropriate credit to the original author(s) and the source, provide a link to the Creative Commons licence, and indicate if changes were made. The images or other third party material in this article are included in the article's Creative Commons licence, unless indicated otherwise in a credit line to the material. If material is not included in the article's Creative Commons licence and your intended use is not permitted by statutory regulation or exceeds the permitted use, you will need to obtain permission directly from the copyright holder. To view a copy of this licence, visit http://creativecommons.org/licenses/by/4.0/.

\section{References}

Ajjawi, R., \& Higgs, J. (2012). Core components of communication of clinical reasoning: a qualitative study with experienced Australian physiotherapists. Advances in Health Sciences Education, 17(1), 107-119. https://doi.org/10.1007/s10459-011-9302-7.

Araújo, D., Davids, K., \& Hristovski, R. (2006). The ecological dynamics of decision making in sport. Psychology of Sport and Exercise, 7(6), 653-676. https://doi.org/10.1016/j.psychsport.2006.07.002.

Araújo, D., Hristovski, R., Seifert, L., Carvalho, J., \& Davids, K. (2017). Ecological cognition: expert decision-making behaviour in sport. International Review of Sport and Exercise Psychology, 1-25. https://doi.org/10.1080/1750984X.2017.1349826.

Arocha, J. F., Wang, D., \& Patel, V. L. (2005). Identifying reasoning strategies in medical decision making: a methodological guide. Journal of Biomedical Informatics, 38(2), 154-171. https://doi.org/10.1016/j.jbi. 2005.02.001.

Assaraf, O. B.-Z., \& Orion, N. (2009). System thinking skills at the elementary school level. Journal of Research in Science Teaching, 540-563. https://doi.org/10.1002/tea.20351.

Baber, C., Chemero, T., \& Hall, J. (2019). What the Jeweller's hand tells the Jeweller's brain: tool use, creativity and embodied cognition. Philosophy \& Technology, 32(2), 283-302. https://doi.org/10.1007/ s13347-017-0292-0.

Becker, R. O., Reichmanis, M., Marino, A. A., \& Spadaro, J. A. (1976). Electrophysiological correlates of acupuncture points and meridians. Psychoenergetic Systems, 1, 105-112.

Bell, I. R., Koithan, M., \& Pincus, D. (2012). Methodological implications of nonlinear dynamical systems models for whole systems of complementary and alternative medicine. Forschende Komplementärmedizin / Research in Complementary Medicine, 19(suppl 1), 15-21. https://doi.org/10. $1159 / 000335183$.

Beresford-Cooke, C. (2003). Shiatsu. Grundlagen und Praxis. Urban \& Fischer.

Bickhard, M. H., \& Terveen, L. (1995). Foundational issues in artificial intelligence and cognitive science: Impasse and solution. Elsevier.

Blackburn, J., \& Price, C. (2007). Implications of presence in manual therapy. Journal of Bodywork and Movement Therapies, 11(1), 68-77. https://doi.org/10.1016/j.jbmt.2006.05.002.

Braude, H. D. (2017). Skilled know-how, virtuosity, and expertise in clinical practice. In T. Schramme \& S. Edwards (Eds.), Handbook of the Philosophy of Medicine (pp. 699-716). Netherlands: Springer. https:// doi.org/10.1007/978-94-017-8688-1_68.

Brehmer, B. (1992). Dynamic decision making: human control of complex systems. Acta Psychologica, 81(3), 211-241. https://doi.org/10.1016/0001-6918(92)90019-A.

Cisek, P., \& Kalaska, J. F. (2010). Neural mechanisms for interacting with a world full of action choices. Annual Review of Neuroscience, 33(1), 269-298. https://doi.org/10.1146/annurev.neuro.051508.135409.

Clark, A. (1997). Being there: Putting brain, body, and world together again. MIT Press.

Clark, A. (2008). Supersizing the mind. Embodiment, action, and cognitive extension. Oxford: UP.

Clark, A., \& Grush, R. (1999). Towards a cognitive robotics. Adaptive Behavior, 7(1), 5-16. 
Clark, A., \& Toribio, J. (1994). Doing without representing? Synthese, 101(3), 401-431.

Cohen, M. S., Freeman, J. T., \& Wolf, S. (1996). Metarecognition in time-stressed decision making: recognizing, critiquing, and correcting. Human Factors: The Journal of the Human Factors and Ergonomics Society, 38(2), 206-219.

Cowley, S., \& Vallée-Tourangeau, F. (2010). Thinking in action. AI \& Society, 25(4), 469-475. https://doi. org/10.1007/s00146-010-0269-3.

Croskerry, P. (2009a). A universal model of diagnostic reasoning. Academic Medicine, 84(8), 1022-1028.

Croskerry, P. (2009b). Clinical cognition and diagnostic error: applications of a dual process model of reasoning. Advances in Health Sciences Education, 14, 27-35. https://doi.org/10.1007/s10459-0099182-2.

Custers, E. J. F. M. (2013). Medical education and cognitive continuum theory: an alternative perspective on medical problem solving and clinical reasoning. Academic Medicine, 88(8), 1074-1080. https://doi.org/ 10.1097/ACM.0b013e31829a3b10.

Davis, N., Hsiao, C.-P., Popova, Y., \& Magerko, B. (2015). An enactive model of creativity for computational collaboration and co-creation. In N. Zagalo \& P. Branco (Eds.), Creativity in the digital age (pp. 109133). London: Springer. https://doi.org/10.1007/978-1-4471-6681-8_7.

Dawson, M. R. W., Dupuis, B., \& Wilson, M. (2010). From bricks to brains. The embodied cognitive science of Lego Robots. AU Press.

de Bruin, L. C., \& Kästner, L. (2012). Dynamic embodied cognition. Phenomenology and the Cognitive Sciences, 11(4), 541-563. https://doi.org/10.1007/s11097-011-9223-1.

Di Paolo, E., \& DeJaegher, H. (2007). Participatory sense-making: an enactive approach to social cognition. Phenomenology and the Cognitive Sciences, 6(4), 485-507. https://doi.org/10.1007/s11097-007-9076-9.

Di Paolo, E. A., Rohde, M., \& Iizuka, H. (2008). Sensitivity to social contingency or stability of interaction? Modelling the dynamics of perceptual crossing. New Ideas in Psychology, 26(2), 278-294. https://doi.org/ 10.1016/j.newideapsych.2007.07.006.

Di Paolo, E. A., Buhrmann, T., \& Barandiaran, X. E. (2017). Sensorimotor life: An enactive proposal (first edition). Oxford University Press.

Dumas, G., Laroche, J., \& Lehmann, A. (2014). Your body, my body, our coupling moves our bodies. Frontiers in Human Neuroscience, 8, 1004. https://doi.org/10.3389/fnhum.2014.01004.

Edwards, I., Jones, M., Carr, J., Braunack-Mayer, A., \& Jensen, G. M. (2004). Clinical reasoning strategies in physical therapy. Physical Therapy, 84(4), 312-330.

Eliasmith, C. (2012). The complex systems approach: rhetoric or revolution. Topics in Cognitive Science, 4(1), 72-77. https://doi.org/10.1111/j.1756-8765.2011.01169.x.

Endsley, M. R. (1995). Toward a theory of situation awareness in dynamic systems. Human Factors: The Journal of the Human Factors and Ergonomics Society, 37(1), 32-64.

Engel, A. K. (2010). Directive minds: How dynamics shapes cognition. Enaction: Towards a new paradigm for cognitive science, 219-243.

Engel, A. K., Maye, A., Kurthen, M., \& König, P. (2013). Where's the action? The pragmatic turn in cognitive science. Trends in Cognitive Sciences, 17(5), 202-209. https://doi.org/10.1016/j.tics.2013.03.006.

Ericsson, K. A., \& Kintsch, W. (1995). Long-term working memory. Psychological Review, 102(2), 211-245.

Esteves, P. T., de Oliveira, R. F., \& Araújo, D. (2011). Posture-related affordances guide attacks in basketball. Psychology of Sport and Exercise, 12(6), 639-644. https://doi.org/10.1016/j.psychsport.2011.06.007.

Fajen, B. R., Riley, M. A., \& Turvey, M. T. (2009). Information, affordances, and the control of action in sport. International Journal of Sport Psychology, 40(1), 79.

Fogel, A. (1993). Developing through relationships. Origins of communication, self, and culture. The University of Chicago Press.

Fogel, A. (2013). Better or worse: a study of day-to-day changes over five months of Rosen method bodywork treatment for chronic low back pain. International Journal of Therapeutic Massage \& Bodywork, 6(3), 14-24.

Fuchs, T., \& DeJaegher, H. (2009). Enactive intersubjectivity: participatory sense-making and mutual incorporation. Phenomenology and the Cognitive Sciences, 8(4), 465-486. https://doi.org/10.1007/ s11097-009-9136-4.

Gallagher, S. (2017). Enactivist interventions. Rethinking the Mind. Oxford University Press.

Gaver, W. (1991). Technology affordances. In S. P. Robertson, G. M. Olson, J. S. Olson (Eds.), Proceedings of the ACM CHI 91 Human Factors in Computing Systems Conference April 28-June 5 (pp. 79-84).

Gesbert, V., Durny, A., \& Hauw, D. (2017). How do soccer players adjust their activity in team coordination? An enactive phenomenological analysis. Frontiers in Psychology, 8, 854. https://doi.org/10.3389/fpsyg. 2017.00854 . 
Gonzalez, C., Vanyukov, P., \& Martin, M. K. (2005). The use of microworlds to study dynamic decision making. Computers in Human Behavior, 21(2), 273-286. https://doi.org/10.1016/j.chb.2004.02.014.

Gonzalez, C., Fakhari, P., \& Busemeyer, J. (2017). Dynamic decision making: learning processes and new research directions. Human Factors: The Journal of the Human Factors and Ergonomics Society, 59(5), 713-721. https://doi.org/10.1177/0018720817710347.

Grace, S., Orrock, P., Vaughan, B., Blaich, R., \& Coutts, R. (2016). Understanding clinical reasoning in osteopathy: a qualitative research approach. Chiropractic \& Manual Therapies, 24(1), 6. https://doi.org/ 10.1186/s12998-016-0087-x.

Grush, R. (2004). The emulation theory of representation: motor control, imagery, and perception. Behavioral and Brain Sciences, 27(03), 377-396. https://doi.org/10.1017/S0140525X04000093.

Haken, H., \& Schiepek, G. (2010). Synergetik in der Psychologie: Selbstorganisation verstehen und gestalten. Hogrefe.

Herfel, W., Rodrigues, D., \& Gao, Y. (2007). Chinese medicine and the dynamic conceptions of health and disease. Journal of Chinese Philosophy, 34, 57-79. https://doi.org/10.1111/j.1540-6253.2007.00453.x.

Higgs, J., \& Jones, M. A. (2000). Clinical decision making and multiple problem spaces. In Clinical reasoning in the health professions (pp. 3-17). Elsevier.

Hotaling, J. M., Fakhari, P., \& Busemeyer, J. R. (2015). Dynamic decision making. In International encyclopedia of the social \& behavioral sciences (pp. 708-713). Elsevier. https://oi.org/10.1016/ B978-0-08-097086-8.43040-0.

Hsu, E. (1999). The transmission of Chinese medicine. Cambridge University Press.

Ihde, D., \& Malafouris, L. (2019). Homo faber revisited: postphenomenology and material engagement theory. Philosophy \& Technology, 32(2), 195-214. https://doi.org/10.1007/s13347-018-0321-7.

Ingold, T. (2015). The life of lines. Routledge.

Jacobson, M. J. (2000). Problem solving about complex systems: Differences between experts and novices. In B. Fishman \& S. O'Connor-Divelbiss (Eds.), Fourth International Conference of the Learning Sciences (pp. 14-21). Erlbaum.

Jacobson, M. J., \& Wilensky, U. (2006). Complex systems in education: scientific and educational importance and implications for the learning sciences. Journal of the Learning Sciences, 15(1), 11-34. https://doi.org/ 10.1207/s15327809j1s1501_4.

Jansson, A. (1994). Pathologies in dynamic decision making: consequences or precursors of failure? Sprache \& Kognition, 13(3), 160-173.

Jensen, G. M., Gwyer, J., Shepard, K. F., \& Hack, L. M. (2000). Expert practice in physical therapy. Physical Therapy, 80(1), 28-43.

Kavoussi, B. (2007). Chinese medicine: a cognitive and epistemological review. Evidence-based Complementary and Alternative Medicine, 4(3), 293-298. https://doi.org/10.1093/ecam/nem005.

Kimmel, M. (2017). The complexity of skillscapes: Skill sets, synergies, and meta-regulation in joint embodied improvisation. In J. Gore \& P. Ward (Eds.), Proceedings of the 13th International Conference on Naturalistic Decision Making, 20-23 June 2017 (pp. 102-109).

Kimmel, M., \& Rogler, C. (2019). The anatomy of antagonistic coregulation: emergent coordination, path dependency, and the interplay of parameters in Aikido. Human Movement Science, 63, 231-253.

Kimmel, M., Irran, C., \& Luger, M. (2015). Bodywork as systemic and inter-enactive competence: Participatory process management in Feldenkrais ${ }^{\circledR}$ method \& Zen Shiatsu. Frontiers in Psychology for Clinical Settings, 5, 1424. https://doi.org/10.3389/fpsyg.2014.01424.

Kimmel, M., Hristova, D., \& Kussmaul, K. (2018). Sources of embodied creativity: interactivity and ideation in contact improvisation. Behavioral Sciences, 8(6), 52. https://doi.org/10.3390/bs8060052.

Kirsh, D. (1991). Today the earwig, tomorrow man? Artificial Intelligence, 47, 161-184.

Kirsh, D. (2009). Problem solving and situated cognition. In P. Robbins \& M. Aydede (Eds.), The Cambridge handbook of situated cognition (pp. 264-306). Cambridge UP.

Kirsh, D. (2014). The importance of chance and interactivity in creativity. Pragmatics \& Cognition, 22(1), 526. https://doi.org/10.1075/pc.22.1.01 kir.

Kirsh, D., \& Maglio, P. (1994). On distinguishing epistemic from pragmatic action. Cognitive Science, 18, 513-549.

Kiverstein, J. D., \& Rietveld, E. (2018). Reconceiving representation-hungry cognition: an ecological-enactive proposal. Adaptive Behavior, 26(4), 147-163. https://doi.org/10.1177/1059712318772778.

Klemme, B., \& Siegmann, G. (2015). Clinical reasoning (2nd ed.). Thieme.

Koithan, M., Bell, I. R., Niemeyer, K., \& Pincus, D. (2012). A complex systems science perspective for whole systems of complementary and alternative medicine research. Forschende Komplementärmedizin / Research in Complementary Medicine, 19(suppl 1), 7-14. https://doi.org/10.1159/000335181. 
Lawson, A. E., \& Daniel, E. S. (2011). Inferences of clinical diagnostic reasoning and diagnostic error. Journal of Biomedical Informatics, 44(3), 402-412. https://doi.org/10.1016/j.jbi.2010.01.003.

Liu, T. (2018). The scientific hypothesis of an "energy system" in the human body. Journal of Traditional Chinese Medical Sciences, 5(1), 29-34. https://doi.org/10.1016/j.jtcms.2018.02.003.

Löhr, G. (2019). Embodied cognition and abstract concepts: do concept empiricists leave anything out? Philosophical Psychology, 32(2), 161-185. https://doi.org/10.1080/09515089.2018.1517207.

MacIntyre, T. E., Igou, E. R., Campbell, M. J., Moran, A. P., \& Matthews, J. (2014). Metacognition and action: a new pathway to understanding social and cognitive aspects of expertise in sport. Frontiers in Psychology, 5, 1155. https://doi.org/10.3389/fpsyg.2014.01155.

Macquet, A. C. (2009). Recognition within the decision-making process: a case study of expert volleyball players. Journal of Applied Sport Psychology, 21(1), 64-79. https://doi.org/10.1080/ 10413200802575759.

Malafouris, L. (2013). How things shape the mind. A theory of material engagement. MIT Press.

Marewski, J. N., \& Gigerenzer, G. (2012). Heuristic decision making in medicine. Dialogues in Clinical Neuroscience, 14(1), 77-89.

Monteiro, S. M., \& Norman, G. (2013). Diagnostic reasoning: where we've been, where we're going. Teaching and Learning in Medicine, 25(sup1), S26-S32. https://doi.org/10.1080/10401334.2013. 842911.

Norgaard, M. (2011). Descriptions of improvisational thinking by artist-level jazz musicians. Journal of Research in Music Education, 59(2), 109-127. https://doi.org/10.1177/0022429411405669.

Norman, G., Young, M., \& Brooks, L. (2007). Non-analytical models of clinical reasoning: the role of experience. Medical Education, 41, 1140-1145. https://doi.org/10.1111/j.1365-2923.2007.02914.x.

Normann, B. (2020). Facilitation of movement: new perspectives provide expanded insights to guide clinical practice. Physiotherapy Theory and Practice, 36(7), 769-778. https://doi.org/10.1080/09593985.2018. 1493165.

Øberg, G. K., Normann, B., \& Gallagher, S. (2015). Embodied-enactive clinical reasoning in physical therapy. Physiotherapy Theory and Practice, 31(4), 244-252. https://doi.org/10.3109/09593985.2014.1002873.

Oschman, J. L. (2000). Energy medicine. The scientific basis. Churchill Livingstone.

Palanjian, K. (2004). Shiatsu. Seminars in Integrative Medicine, 2(3), 107-115. https://doi.org/10.1016/j.sigm. 2004.09.001.

Passos, P., Milho, J., Fonseca, S., Borges, J., Araújo, D., \& Davids, K. (2011). Interpersonal distance regulates functional grouping tendencies of agents in team sports. Journal of Motor Behavior, 43(2), 155-163. https://doi.org/10.1080/00222895.2011.552078.

Passos, P., Cordovil, R., Fernandes, O., \& Barreiros, J. (2012). Perceiving affordances in rugby union. Journal of Sports Sciences, 30(11), 1175-1182. https://doi.org/10.1080/02640414.2012.695082.

Patel, V. L., \& Ramoni, M. F. (1997). Cognitive models of directional inference in expert medical reasoning. In P. J. Feltovich, K. M. Ford, \& R. R. Hoffman (Eds.), Expertise in context (pp. 67-99). AAAI Press.

Patel, V. L., Arocha, J. F., \& Zhang, J. (2005). Thinking and reasoning in medicine. In K. J. Holyoak \& R. G. Morrison (Eds.), The Cambridge Handbook of Thinking and Reasoning (pp. 726-750). Cambridge University Press.

Patel, V. L., Arocha, J. F., Zhang, J. (2012). Medical reasoning and thinking. In K. J. Holyoak \& R. G. Morrison (Eds.), The Oxford Handbook of Thinking and Reasoning (pp. 736-754). Oxford University Press.

Pedersen, S. B. (2015). The cognitive ecology of human error in emergency medicine. University of Southern Denmark.

Pincus, D. (2012). Self-organizing biopsychosocial dynamics and the patient-healer relationship. Forschende Komplementärmedizin / Research in Complementary Medicine, 19(suppl 1), 22-29. https://doi.org/10. $1159 / 000335186$.

Pincus, D., \& Metten, A. (2010). Nonlinear dynamics in biopsychosocial resilience. Nonlinear Dynamics Psychology \& Life Sciences, 14(4), 353-380.

Poizat, G., Sève, C., \& Saury, J. (2013). Qualitative aspects of performance analysis. In T. McGarry, P. O'Donoghue, J. Sampaio (Eds.), Routledge handbook of sports performance analysis (pp. 309-320). Routledge.

Porkert, M., \& Ullmann, C. (1982). Chinese medicine. MIT Press.

Raczaszek-Leonardi. (2009). Symbols as constraints: the structuring role of dynamics and self-organization in natural language. Pragmatics \& Cognition, 17(3), 653-676. https://doi.org/10.1075/pc.17.3.09ras.

Ramsey, W. M. (2007). Representation reconsidered. Cambridge University Press.

Robbins, P., \& Aydede, M. (2009). The Cambridge handbook of situated cognition. Cambridge UP. 
Robinson, N., Lorenc, A., \& Liao, X. (2011). The evidence for Shiatsu: a systematic review of Shiatsu and acupressure. BMC Complementary and Alternative Medicine, 11, 88. https://doi.org/10.1186/1472-688211-88

Runeson, S. (1977). On the possibility of "smart" perceptual mechanisms. Scandinavian Journal of Psychology, 18, 172-179.

Schön, D. (1991). The reflective practitioner. Ashgate Publishing Ltd.

Schoppek, W. (2002). Examples, rules, and strategies in the control of dynamic systems. Cognitive Science Quarterly, 2(1), 63-92.

Seifert, L., Button, C., \& Davids, K. (2013). Key properties of expert movement systems in sport: an ecological dynamics perspective. Sports Medicine, 43(3), 167-178. https://doi.org/10.1007/s40279-0120011-z.

Sibbald, M., \& de Bruin, A. B. H. (2012). Feasibility of self-reflection as a tool to balance clinical reasoning strategies. Advances in Health Sciences Education, 17(3), 419-429. https://doi.org/10.1007/s10459-0119320-5.

Silberstein, M., \& Chemero, A. (2011). Dynamics, agency and intentional action. Humana Mente, 15, 1-19.

Silva, P. L., Fonseca, S. T., \& Turvey, M. T. (2010). Is tensegrity the functional architecture of the equilibrium point hypothesis. Motor Control, 14(3), e35-e40.

Silva, P., Garganta, J., Araújo, D., Davids, K., \& Aguiar, P. (2013). Shared knowledge or shared affordances? Insights from an ecological dynamics approach to team coordination in sports. Sports Medicine, 43(9), 765-772. https://doi.org/10.1007/s40279-013-0070-9.

Smith, L. (2010). More than concepts. In D. Mareschal, P. C. Quinn, \& E. G. Lea Stephen (Eds.), Making human concepts (pp. 335-364). Oxford UP.

Steffensen, S. V. (2013). Human interactivity: Problem-solving, solution-probing and verbal patterns in the wild. In S. J. Cowley \& F. Vallée-Tourangeau (Eds.), Cognition Beyond the Brain (pp. 195-221). Springer London. http://link.springer.com/10.1007/978-1-4471-5125-8_11

Steffensen, S. V., Vallée-Tourangeau, F., \& Vallée-Tourangeau, G. (2016). Cognitive events in a problemsolving task: a qualitative method for investigating interactivity in the 17 animals problem. Journal of Cognitive Psychology, 28(1), 79-105. https://doi.org/10.1080/20445911.2015.1095193.

Steiner, S., Macquet, A.-C., \& Seiler, R. (2017). An integrative perspective on interpersonal coordination in interactive team sports. Frontiers in Psychology, 8,1440. https://doi.org/10.3389/fpsyg.2017.01440.

Sterman, J. D. (2000). Business Dynamics: Systems thinking and modeling for a complex world. Irwin/ McGraw-Hill.

Suchman, L. A. (1986). Plans and situated actions. The problem of human machine communication. Cambridge University Press.

Torrance, S., \& Schumann, F. (2019). The spur of the moment: what jazz improvisation tells cognitive science. AI \& Society 34, 251-268. https://doi.org/10.1007/s00146-018-0838-4

Torrents, C., Hristovski, R., Coterón, J., \& Ric, A. (2016). Interpersonal coordination in contact improvisation dance. In P. Passos, K. Davids, \& J. Y. Chow (Eds.), Interpersonal coordination and performance in social systems (pp. 94-108). Routledge.

Trasmundi, S. B., \& Linell, P. (2017). Insights and their emergence in everyday practices: the interplay between problems and solutions in emergency medicine. Pragmatics \& Cognition, 24(1), 62-90. https:// doi.org/10.1075/pc.17002.tra.

Trasmundi, S. B., \& Steffensen, S. V. (2016). Meaning emergence in the ecology of dialogical systems. Psychology of Language and Communication, 20(2), 154-181. https://doi.org/10.1515/plc-2016-0009.

Trevarthen, C. (1979). Communication and cooperation in early infancy: A description of primary intersubjectivity. In M. Bullowa (Ed.), Before speech (pp. 321-347). Cambridge UP.

Tschacher, W., Scheier, C., \& Grawe, K. (1998). Order and pattern formation in psychotherapy. Nonlinear Dynamics, Psychology, and Life Sciences, 2(3), 195-215.

Tschacher, W., Dauwalder, J.-P., \& Haken, H. (2003). Self-organizing systems show apparent intentionality. In W. Tschacher \& J.-P. Dauwalder (Eds.), The Dynamical Systems Approach to Cognition (pp. 183200). World Scientific.

Veenman, M. V. J., Van Hout-Wolters, B. H. A. M., \& Afflerbach, P. (2006). Metacognition and learning: conceptual and methodological considerations. Metacognition and Learning, 1(1), 3-14. https://doi.org/ 10.1007/s11409-006-6893-0.

Walton, A. E., Richardson, M. J., Langland-Hassan, P., \& Chemero, A. (2015). Improvisation and the selforganization of multiple musical bodies. Frontiers in Psychology, 06, 313. https://doi.org/10.3389/fpsyg. 2015.00313 .

Ward, D., Silverman, D., \& Villalobos, M. (2017). Introduction: the varieties of enactivism. Topoi, 36(3), 365-375. https://doi.org/10.1007/s11245-017-9484-6. 
Weiss, J. N., Qu, Z., \& Garfinkel, A. (2003). Understanding biological complexity: lessons from the past. The FASEB Journal, 17(1), 1-6. https://doi.org/10.1096/fj.02-0408rev.

Wheeler, M. (2005). Reconstructing the cognitive world. MIT Press.

Zhang, J., Patel, V. L., Johnson, T. R., \& Shortliffe, E. H. (2004). A cognitive taxonomy of medical errors. Journal of Biomedical Informatics, 37(3), 193-204. https://doi.org/10.1016/j.jbi.2004.04.004.

Zwaan, R. A. (1999). Embodied cognition, perceptual simulation, and situation models. Discourse Processes, $28(1), 81-88$.

Publisher's note Springer Nature remains neutral with regard to jurisdictional claims in published maps and institutional affiliations. 